\title{
Questionário Reduzido do Estilo Pessoal do Terapeuta: evidências de validade
}

\author{
Emanuel M. Silva Palma¹, Sônia Maria Guedes Gondim \\ Universidade Federal da Bahia, Salvador-BA, Brasil
}

\section{RESUMO}

O estilo pessoal do terapeuta refere-se a um construto multidimensional composto por cinco funções básicas presentes em seu processo de trabalho. O objetivo deste estudo foi analisar a validade fatorial da versão abreviada do Questionário Reduzido do Estilo Pessoal do Terapeuta (no original, Estilo Personal del Terapeuta-Cuestionario - EPT-C) em uma amostra de psicoterapeutas brasileiros. Uma análise fatorial confirmatória foi conduzida em uma amostra de 674 psicoterapeutas, sendo testados os modelos penta e tetrafatorial. O modelo tetrafatorial apresentou melhores índices de ajuste aos dados, e foram encontrados valores aceitáveis de consistência interna para suas dimensões. Examinou-se também a capacidade de o instrumento detectar diferenças estilísticas entre terapeutas em função de sua orientação teórica. De forma geral, terapeutas das orientações psicanalítica, humanista-existencial, analítica e sistêmica compõem um grupo mais homogêneo do que os terapeutas cognitivos e analítico-comportamentais quanto às funções do EPT-C. Discutem-se os ajustes realizados ao modelo, as diferenças intergrupais encontradas e sugestões para revisão do modelo.

Palavras-chave: psicoterapeuta; validade estatística; personalidade.

\section{ABSTRACT - Therapist Personal Style Short Questionnaire: Validity evidence}

Therapist's personal style refers to a multidimensional construct consisting of five basic functions underlying the therapist's work process. The objective of this article is to analyze the factor validity of the Cuestionario del Estilo del Terapeuta (EPT-C) in a sample of Brazilian psychotherapists. Participants in the study were 674 psychotherapists. A confirmatory factor analysis was conducted to test a five-factor model and a four-factor model. The four-factor model showed better fit to the data, and acceptable internal reliability values were found for its dimensions. Furthermore, we examined the instrument's ability to detect stylistic differences among groups of therapists as a function of their theoretical orientation. In general, psychoanalysts, humanistic-existential, analytical and systemic therapists consisted of a more homogeneous group than cognitive therapists and behavior therapists. The model fitting process, intergroup differences and suggestions for the revision of the model are discussed.

Keywords: psychotherapist; statistical validity; personality.

\section{RESUMEN - Cuestionario del Estilo Personal del Terapeuta Versión Abreviada: evidencias de validez}

El estilo personal del terapeuta se refiere a un constructo multidimensional compuesto por cinco funciones básicas presentes en su proceso de trabajo. El objetivo de este estudio fue analizar la validez factorial de la versión abreviada del Cuestionario del Estilo Personal del Terapeuta (EPT-C) en una muestra de psicoterapeutas brasileños. Un análisis factorial confirmatorio se realizó sobre una muestra de 674 psicoterapeutas, y probó los modelos penta y tetrafactorial. El modelo tetrafactorial mostró mejores índices de ajuste a los datos y niveles aceptables de consistencia interna de sus dimensiones. Además, se examinó la capacidad del instrumento en detectar diferencias de estilo entre los terapeutas de distintas orientaciones teóricas. En general, los terapeutas psicoanalíticos, humanista-existenciales, analíticos y sistémicos forman un grupo más homogéneo que los terapeutas cognitivos y analítico-comportamentales con relación a las funciones del EPT-C. Se discuten los ajustes realizados en los modelos, las diferencias entre los grupos y sugerencias para la revisión del modelo. Palabras clave: psicoterapeuta; validez estadística; personalidad.

O campo de pesquisas em psicoterapia está repleto de investigações sobre os atributos pessoais do terapeuta e as técnicas utilizadas durante a sessão (e.g., traços de personalidade, autoestima, motivação, visão de mundo, bem como exploração, facilitação e reflexão de sentimentos do paciente) que contribuem para o sucesso do tratamento de transtornos psicológicos (Baldwin \& Imel, 2013; Heinonen et al., 2014; Schöttke, Flückiger, Goldberg, Eversmann, \& Lange, 2015). Independentemente da abordagem teórica adotada pelo terapeuta, esses atributos e procedimentos parecem ter um impacto positivo não só sobre os resultados do tratamento, como também sobre a saúde e o bem-estar pessoal do profissional 
(Råbu, Moltu, Binder, \& McLeod, 2015). Tendo em vista que parte considerável do sucesso do processo terapêutico pode ser atribuída a características pessoais do profissional (Baldwin \& Imel, 2013), estudos recentes têm sido conduzidos para esclarecer quais variáveis do terapeuta contribuem para o exercício de seu trabalho e, em alguma medida, para sua qualidade de vida. Nesse sentido, alguns autores têm demonstrado crescente interesse teórico e empírico em estudar os estilos do terapeuta, dentre os quais se encontram o estilo de apego (Degnan, Seymour-Hyde, Harris, \& Berry, 2016), o estilo epistêmico (Lee, Neimeyer, \& Rice, 2013) e o estilo pessoal (Castañeiras, Ledesma, García, \& Fernández-Álvarez, 2008). Neste artigo, o foco recai sobre o estilo pessoal do terapeuta.

Estilo, na perspectiva de Fernández-Álvarez, García, Lo Bianco e Santomá (2003), refere-se àquelas funções do comportamento humano que demonstram, simultaneamente, estabilidade temporal e capacidade de adaptação às condições ambientais, permitindo que o indivíduo possa se reconhecer e ser reconhecido pelos outros. Ao abordar questões estilísticas na prática clínica, Castañeiras et al. (2008) defendem que o estilo pessoal do terapeuta é um componente básico de todo processo terapêutico (de natureza multidimensional), sendo, pois, composto por um conjunto de funções conduzidas de forma articulada. Essas funções expressam disposições, riscos e atitudes que o terapeuta põe em prática ao conduzir seu trabalho (Oliveira, Nunes, Fernández-Álvarez, \& García, 2006). Para alguns autores (Fernandez-Álvarez et al., 2003; Oliveira et al., 2006), o estilo pessoal do terapeuta resulta da interação entre variáveis de três categorias, a saber:

1. posição socioprofissional;

2. história de vida, traços de personalidade, atitudes e visão de mundo; e

3. modos dominantes de comunicação.

Esses autores defendem também que o estilo pode funcionar como uma variável moderadora da relação entre as técnicas utilizadas e os resultados de tratamentos conduzidos pelo terapeuta (Fernández-Álvarez et al., 2003). Nesse contexto, cabe ressaltar que não existe uma combinação ideal de padrões estilísticos do terapeuta. Demandas situacionais específicas (e.g., características individuais do paciente, tipos de transtornos e condições de trabalho) podem requerer diferentes ações por parte do terapeuta para um desfecho clínico positivo.

De acordo com Fernández-Álvarez et al. (2003), o estilo pessoal do terapeuta é constituído por cinco funções básicas que se apresentam como um continuum bipolar (Tabela 1). Mediante a realização de uma série de estudos transculturais com amostras de terapeutas de diferentes orientações teóricas, essas dimensões têm sido refinadas do ponto de vista conceitual e empírico. Além disso, elas constituem a base do Estilo Personal del Terapeuta-Cuestionario (EPT-C), Questionário Reduzido do Estilo Pessoal do Terapeuta (em português), instrumento de autorrelato desenvolvido por Fernández-Álvarez et al. (2003) para mensurar esse construto.

O EPT-C tem se mostrado uma promissora medida de aspectos estilísticos do trabalho do terapeuta e tem sido utilizado em diferentes contextos culturais, revelando similaridades e diferenças entre esses profissionais em função de variáveis como gênero, tempo de experiência, orientação teórica, traços de personalidade e uso de técnicas psicoterápicas (Lee et al., 2013; Oliveira et al., 2006). Tanto em sua versão original (36 itens) quanto em sua versão reduzida (21 itens), o EPT-C tem apresentado bons indicadores psicométricos. $\mathrm{Na}$ sua versão original, a consistência interna, calculada pelo alfa de Cronbach, variou de 0,69 a 0,80, e a validade temporal teste-reteste, com base na correlação de Pearson, revelou valores situados entre 0,76 e 0,82 para as 5 dimensões (Fernández-Álvarez et al., 2003). Na sua versão reduzida, foram obtidos valores de alfa considerados bons para todas as dimensões, variando de 0,60 a 0,75 (Castañeiras et al., 2008). Observa-se, no entanto, que a dimensão "Instrução" tem sido a responsável pelos menores valores de confiabilidade em alguns estudos, levando alguns autores a questionarem a sua pertinência à estruturação do modelo e também a sua validade de construto (Carvalho, Corbella, \& Matos, 2011; Oliveira et al., 2006).

O EPT-C apresenta alta correlação entre suas diferentes dimensões. Isso sugere que o estilo do terapeuta pode também ser investigado a partir de dois domínios,

Tabela 1

Funções, características e dimensões do estilo pessoal do terapeuta

\begin{tabular}{lrc}
\hline Funções & Características & Dimensões bipolares \\
\hline Atenção & Seleção das informações relevantes à condução do processo terapêutico. & Aberta - Focada \\
Envolvimento & Envolvimento do terapeuta com o trabalho. & Baixo grau - Alto grau \\
Expressão & Modo de se comunicar afetivamente com os pacientes. & Distante - Próxima \\
Instrução & Estabelecimento e regulação do setting terapêutico (e.g., horário, pagamento). & Rígida - Flexível \\
Operação & Implementação instrumental (e.g., procedimentos e técnicas). & Livre - Planejada \\
\hline
\end{tabular}

Fonte: Fernández-Álvarez et al. (2003). 
ou fatores, de ordem superior: um técnico-cognitivo, composto pelas funções "Atenção" e "Operação", e outro afetivo-motivacional, composto pelas funções "Expressão" e "Envolvimento". Por exemplo, em um estudo conduzido por Fernández-Álvarez et al. (2003), uma atenção aberta e uma operação livre demonstram um alto grau de correlação entre si, enquanto uma expressão mais próxima correlacionou-se fortemente com um maior grau de envolvimento.

Outros estudos têm sido conduzidos para testar a validade do construto por meio do uso de diferentes técnicas. Por exemplo, ao testar o modelo reduzido em uma amostra de terapeutas argentinos mediante uma análise fatorial confirmatória, Castañeiras et al. (2008) encontraram bons índices de ajuste entre o modelo de medida de 5 fatores e as dimensões teóricas (Comparative Fit Index-CFI=0,91; Goodness-of-Fit Index - GFI=0,93; Root Mean Square Error of Approximation RMSEA =0,05; Incremental Fit Index - IFI=0,91). Além disso, descobriram correlações significativas entre os fatores "Atenção" e "Operação" $(r=0,75 ; p<0,01)$ e "Envolvimento" e "Expressão" $(r=0,25 ; p<0,01)$. Resultados semelhantes foram encontrados em uma amostra de terapeutas portugueses (Carvalho et al., 2011). Nesse estudo, também foram encontrados índices aceitáveis para o teste do modelo da medida abreviada $(\mathrm{CFI}=0,90 ; \mathrm{RMSEA}=0,045)$. Também encontraram consideráveis correlações entre os fatores "Atenção" e "Operação" $(r=0,68 ; p<0,01)$ e "Envolvimento" e "Expressão" $(r=0,52 ; p<0,01)$. Os achados das análises confirmatórias reforçam a associação significativa entre os fatores "Atenção" e "Operação" e "Envolvimento" e "Expressão". Por outro lado, reafirma-se que a dimensão "Instrução" tem se comportado de modo menos estável, alcançando valores mais baixos de consistência interna e apresentando fracas correlações com as demais dimensões.

No contexto brasileiro, Oliveira et al. (2006) apresentaram alguns dados preliminares da versão original do EPT-C. Nesse estudo, foram apresentados os procedimentos de adaptação do instrumento, os índices de consistência interna de suas dimensões e os resultados de algumas análises. Entretanto, os autores não relataram o uso de análises fatoriais exploratórias ou confirmatórias do modelo. Os alfas de Cronbach variaram de 0,52 a 0,77 para as 5 dimensões, sendo que os valores mais baixos foram os das dimensões "Expressão" $(\alpha=0,55)$ e "Instrução" $(\alpha=0,52)$. As outras dimensões obtiveram valores acima de 0,70 , sendo considerados bons. Os autores observaram ainda que, em relação aos anos de experiência, apenas a dimensão "Atenção" foi significativamente diferente entre os grupos, sendo que os terapeutas menos experientes tenderam a apresentar uma atenção mais focada do que os mais experientes. Terapeutas do sexo masculino apresentaram atenção mais focada do que os do sexo feminino. Quanto às orientações teóricas (e.g., psicanalítica, cognitiva e integrativa), foram observadas diferenças significativas em todas as dimensões, resultados semelhantes àqueles encontrados no estudo de Fernández-Álvarez et al. (2003). Oliveira et al. (2006) descobriram, por exemplo, que psicoterapeutas de orientação psicanalítica, em seu conjunto, tenderam a ter uma atenção mais aberta e um modo de operação mais livre, além de apresentarem escores mais baixos na função expressiva; em termos de envolvimento, demonstraram menor grau, se comparados aos terapeutas cognitivos e integrativos.

De modo geral, o EPT-C tem se mostrado um instrumento com boas qualidades psicométricas, sendo capaz de detectar diferenças estilísticas conforme as abordagens teóricas adotadas pelos profissionais. Além disso, algumas de suas dimensões parecem sofrer influência de variáveis como gênero e tempo de experiência (Carvalho et al., 2011; Fernández-Álvarez et al., 2003; Oliveira et al., 2006). Nesse sentido, estudos em diferentes contextos culturais e com grupos maiores de psicoterapeutas de diferentes abordagens podem permitir o refinamento teórico-conceitual da medida, fortalecendo seus indicadores de validade, além de apontar para possíveis elementos culturais que podem influenciar os elementos estilísticos do exercício da psicoterapia. Ao ter em conta esse cenário, o objetivo deste estudo foi analisar o ajuste do modelo da estrutura reduzida do questionário EPT-C (21 itens; Castañeiras et al., 2008) em uma amostra de psicoterapeutas brasileiros. Além disso, também foram investigadas as diferenças estilísticas entre terapeutas em função de sua orientação teórica e tempo de experiência.

\section{Método}

\section{Participantes}

Participaram do estudo 674 psicoterapeutas, dos quais $88,6 \%(n=597)$ se identificaram como psicólogos, 7,3\% ( $n=49)$ como médicos e 4,2\% $(n=28)$ como "outros" (e.g., assistente social, enfermeiro e pedagogo). A maioria era do sexo feminino, $78,5 \%(n=529)$, com idade média de 42 anos $(\mathrm{DP}=13,06)$, variando de 22 a 78 anos. Em relação ao local da prática, a maioria, $92 \%(n=620)$, trabalhava em consultório particular, enquanto $4,5 \%(n=30)$ e $3,3 \%(n=20)$ atuavam profissionalmente em hospitais e Centros de Atenção Psicossocial (CAPs), respectivamente. O restante indicou trabalhar em outros dispositivos, como centros comunitários e clínicas-escola. Em relação ao tempo de experiência, houve uma variação de 1 a 50 anos $(M=12,84 ; D P=11)$. Vinte e um psicoterapeutas relataram atuar há menos de um ano quando da realização da coleta. Os psicoterapeutas trabalhavam de $1 \mathrm{a}$ 40 horas semanais $(M=23,49 ; D P=13,9)$ e indicaram 
como orientações teóricas predominantes: psicanálise, $25,2 \%(n=170)$; análise do comportamento, $19,6 \%$ $(n=132)$; cognitiva, $18,2 \% \quad(n=123)$; humanista-existencial, 13,4\% $(n=90)$; analítica, 11,4\% $(n=77)$; sistêmica, $7,4 \%(n=50)$; e outra, $4,8 \%(n=32)$. Outras orientações citadas incluíram psicodrama, esquizoanálise e integrativa.

\section{Instrumentos}

Foi utilizado o EPT-C em sua versão reduzida, composta por 5 dimensões e 21 itens: "Atenção" (quatro itens; e.g.: "Gosto de me sentir surpreendido pelo material do paciente sem ter ideias prévias"); "Envolvimento" (quatro itens; e.g.: "Fora das sessões, tenho em mente os problemas dos pacientes"); "Expressão" (cinco itens, e.g.: "Uma alta proximidade emocional é imprescindível para mudanças terapêuticas"); "Instrução" (três itens; e.g.: "Sou bastante flexível com os horários"); e Operação (cinco itens; e.g.: "Prefiro tratamentos onde estão programados todos os passos a seguir"). Essa versão foi proposta por Castañeiras et al. (2008) e obteve bons índices de ajuste $(\mathrm{CFI}=0,91 ; \mathrm{GFI}=0,93$; RMSEA=0,05; $\mathrm{IFI}=0,91)$ em uma amostra de 486 psicoterapeutas argentinos. Também foi testada, por Carvalho et al. (2011), em uma amostra de psicoterapeutas portugueses, obtendo índices considerados bons após a nova especificação do modelo com base em alguns índices de modificação.

Para a versão brasileira reduzida do instrumento, os 21 itens propostos por Castañeiras et al. (2008) para a versão reduzida em espanhol foram retirados do questionário original adaptado, no Brasil, por Oliveira et al. (2006). Compuseram cada dimensão os seguintes itens do questionário original: "Atenção" $(1,18,33$ e 36), "Envolvimento" (13, 21, 23 e 24), "Expressão" (6, 11, 15, 28 e 29), "Instrução" (9, 27 e 35) e "Operação" (3, 10, 22, 25 e 31). O questionário adaptado por Oliveira et al. (2006) já havia passado pelo processo de tradução, retrotradução e validação semântica por juízes. O questionário reduzido utilizado no presente estudo encontra-se no Apêndice A. Ainda assim, optou-se por comparar os itens extraídos do questionário original adaptado ao português brasileiro às versões reduzidas espanhola e portuguesa, a fim de verificar a sua qualidade semântica e de adaptação. Como alguns itens ainda dificultavam a compreensão, os autores da medida original foram consultados para esclarecer o significado de alguns termos que apresentavam sentido ambíguo. Por fim, o questionário reduzido usado no presente estudo foi aplicado em uma amostra de dez psicoterapeutas de diferentes orientações teóricas. Esses profissionais fizeram comentários relacionados à clareza, à compreensão e à adequação dos itens. Com base em observações dos profissionais, consultoria aos autores da medida original, comparações entre as versões reduzidas em espanhol e português de Portugal e a versão original em português brasileiro, chegou-se à versão final reduzida utilizada neste estudo. Os ajustes finais tiveram como objetivo tornar os itens mais claros e coerentes com suas dimensões. Por exemplo, o item "Tenho em mente os problemas dos pacientes fora das sessões" foi modificado para "Fora das sessões, tenho em mente os problemas dos pacientes". De acordo com alguns terapeutas participantes do teste piloto, a primeira formulação permitia dupla interpretação:

1. o terapeuta pensa nos problemas que o paciente tem fora das sessões, o que pode ser material a ser trabalhado no processo terapêutico;

2. o terapeuta, fora do seu horário de trabalho, pensa nos problemas de seus pacientes.

O item foi reformulado para contemplar com maior clareza o segundo sentido, que era, de fato, o pretendido pelos autores da escala original. Após a realização de todos esses procedimentos, o instrumento foi considerado adequado para uso. Foi também utilizado um questionário sobre dados sociodemográficos e profissionais, como sexo, idade, anos de experiência, dispositivo prevalente de sua prática profissional (e.g., consultório particular, hospital e CAPs) e orientação teórica predominante.

\section{Procedimentos}

Os dados foram coletados por meio de um questionário on-line enviado a profissionais membros de associações e federações nacionais de psicoterapeutas (e.g.: Federação Brasileira de Psicanálise, Associação Brasileira de Terapia Cognitiva e Associação Brasileira de Medicina e Psicoterapia Comportamental). Também foram contatados profissionais por meio de suas páginas web pessoais e de divulgação de serviços. Após lerem o Termo de Consentimento Livre e Esclarecido (TCLE) e concordarem em participar da pesquisa, os participantes respondiam aos itens on-line. O link contendo o questionário contou com 1.233 visitas, sendo que $674(54,9 \%)$ psicoterapeutas completaram todo o questionário. É importante ressaltar que o projeto do qual este estudo faz parte foi aprovado pelo Comitê de Ética em Pesquisa da Faculdade Adventista da Bahia (CAAE: 37816214.7.0000.0042).

\section{Análise de dados}

Para avaliar a qualidade psicométrica da medida, a amostra foi inicialmente dividida aleatoriamente em 2 subgrupos (337 participantes em cada) para permitir a validação cruzada. Empregou-se a análise fatorial confirmatória (método de estimação Maximum Likelihood), tendo como índices de aderência do modelo: qui-quadrado/graus de liberdade $\left(\chi^{2} /\right.$ $g l)$; CFI e o GFI, cuja proximidade de 1,0 representa melhor ajuste do modelo (Byrne, 2001). Além disso, calcularam-se o RMSEA, cujos valores abaixo de 0,08 
são considerados aceitáveis, e o Bayesian information criterion (BIC), para comparação dos modelos (Byrne, 2001). Além disso, a fim de identificar diferenças intergrupais entre os terapeutas de distintas orientações teóricas nas dimensões do EPT-C, foi realizada uma análise de variância (ANOVA one-way) e testes post hoc (Scheffe e Games Howell).

\section{Resultados e Discussão}

Por meio da análise fatorial confirmatória, foram especificados, na primeira amostra, dois modelos para a mensuração do estilo pessoal do terapeuta: um pentafatorial, de acordo com a estrutura proposta por Castañeiras et al. (2008), e outro tetrafatorial, que considerava achados da literatura empírica e índices de modificação. Esse procedimento visou possibilitar a comparação dos índices de ajuste, evidenciando, assim, o melhor modelo. Por fim, para garantir que os ajustes do modelo não se restringissem a uma única amostra, o modelo de melhor ajuste da primeira amostra foi testado na segunda. A Tabela 2 apresenta os índices de ajuste obtidos para os modelos pentafatorial, tetrafatorial e na validação cruzada. É importante observar que, devido à complexidade do modelo, a estrutura pentafatorial teve de ser testada com um número maior de participantes. Optou-se então por testar essa estrutura na amostra geral $(n=674)$.

Como se pode observar, os índices de ajuste do modelo pentafatorial não foram totalmente satisfatórios. Embora o CFI e o RMSEA possam ser considerados bons, o GFI apresentou um valor considerado abaixo do aceitável (Byrne, 2001). Foram verificados os índices de modificação, os indicadores de normalidade (assimetria e curtose - todos considerados adequados), os outliers, de acordo com os valores do Mahalanobis distance $\left(\mathrm{D}^{2}\right)$, e as cargas fatoriais dos itens. Mesmo reespecificando o modelo pentafatorial com base nesses índices (e.g.: estabelecendo um parâmetro de correlação entre os erros dos itens e8 e e9 do fator Operação e eliminando os itens 18 e 15, que apresentaram cargas fatoriais muito baixas), o GFI não apresentou valores mais altos. Ao considerar a literatura empírica sobre a medida, observou-se que a função "Instrução" tem apresentado pouca consistência interna, tendo obtido um baixo valor de alfa de Cronbach em alguns estudos (Carvalho et al., 2011; Oliveira et al., 2006). Além disso, neste estudo, os três itens que compõem esse fator apresentaram as cargas fatoriais mais baixas, variando entre 0,35 e 0,49 . Optou-se então por testar um modelo tetrafatorial nas duas subamostras extraídas aleatoriamente da amostra geral, conservando-se os fatores "Atenção", "Envolvimento", "Expressão" e "Operação". Esse modelo foi inicialmente testado na amostra 1 e apresentou bons índices de ajuste. Verificou-se novamente a baixa carga fatorial (e.g.: $<0,30)$ dos itens 18 e 15 , sendo ambos excluídos do modelo. Esses itens já haviam demonstrado problemas em estudos prévios, como baixa saturação no fator esperado (Castañeiras et al., 2008), o que sugere a sua revisão para melhor refletir a dimensão pretendida.

Depois de realizados esses procedimentos, houve um decréscimo dos valores do $\chi^{2}$, do RMSEA e do BIC, bem como aumento do GFI e do CFI. Essas alterações promoveram uma melhor aderência do modelo aos dados, mantendo os índices dentro dos valores recomendados (Byrne, 2001). Por fim, esse mesmo modelo tetrafatorial foi testado na amostra 2, apresentando índices de ajustamento satisfatórios e semelhantes aos do modelo reespecificado na etapa anterior. Esses índices são bastante semelhantes aos encontrados por Castañeiras et al. (2008), quando testaram a medida reduzida por meio da análise fatorial confirmatória, com GFI e CFI superiores a 0,9 e RMSEA inferior a 0,06 .

Para avaliar a consistência interna das dimensões, optou-se pelo uso do teste confiabilidade composta, sendo considerados desejáveis aqueles valores superiores a 0,70 (Hair, Anderson, Tatham, \& Black, 2005) e aceitáveis aqueles inferiores a esse valor em fases exploratórias da investigação. Obtiveram-se os seguintes valores de confiabilidade composta para os

Tabela 2

Índices de ajustamento dos modelos testados

\begin{tabular}{|c|c|c|c|c|}
\hline \multirow[b]{2}{*}{ Índices de ajustamento } & \multicolumn{3}{|c|}{ Modelos } & \multirow[b]{2}{*}{ Parâmetros } \\
\hline & $\begin{array}{c}\text { Pentafatorial } \\
\text { (amostra total) }\end{array}$ & $\begin{array}{l}\text { Tetrafatorial } \\
\text { (amostra 1) }\end{array}$ & $\begin{array}{c}\text { Tetrafatorial } \\
\text { (amostra 2) }\end{array}$ & \\
\hline$\chi^{2} / g l$ & 3,26 & 1,96 & 1,98 & $<5$ \\
\hline CFI & 0,923 & 0,933 & 0,932 & $\geq 0,90$ \\
\hline GFI & 0,824 & 0,901 & 0,902 & $\geq 0,90$ \\
\hline RMSEA & 0,058 & 0,050 & 0,054 & $\leq 0,08$ \\
\hline BIC & 922,993 & 272,409 & 415,317 & Menor valor \\
\hline
\end{tabular}

Nota: gl=grau de liberdade; CFI=Comparative Fit Index; GFI=Goodness-of-Fit Index; RMSEA=Root Mean Square Error of Approximation; $\mathrm{BIC}=$ Bayesian information criterion. 
quatro fatores: "Atenção" $(0,64)$, "Envolvimento" $(0,64)$, "Expressão" $(0,63)$ e "Operação" $(0,72)$. Além disso, foram calculados os alfas de Cronbach para cada dimensão: "Atenção" $(0,63)$, "Envolvimento" $(0,62)$, "Expressão" $(0,62)$ e "Operação" $(0,71)$. Se comparados aos valores encontrados por Castañeiras et al. (2008) e Carvalho et al. (2011), esses alfas foram ligeiramente inferiores, ainda que considerados aceitáveis.

Como indicador da validade convergente dos fatores, foi calculada a variância extraída média (VEM), cujos valores para os fatores foram: "Atenção" $(0,37)$, "Envolvimento" (0,32), "Expressão" $(0,30)$ e "Operação" (0,34). Embora sejam considerados baixos (Hair et al., 2005), esses valores permitiram a verificação da validade discriminante entre os fatores, comparando as VEMs de cada fator com o quadrado da correlação entre os fatores cuja validade discriminante se pretende avaliar (Marôco, 2014). A Tabela 3 apresenta o quadrado das correlações entre os fatores.

Como se pode observar, não foi possível verificar a validade discriminante entre os fatores "Expressão" e "Atenção" e os fatores "Atenção" e "Operação", pois os valores das VEMs foram inferiores ao quadrado das correlações entre eles. Por exemplo, VEMat $(0,37)$ e VEMex $(0,30)$ são inferiores ao quadrado da correlação entre essas duas dimensões $(0,53)$. As correlações entre esses fatores (Figura 1), aliadas à proximidade com que se articulam esses elementos do estilo do terapeuta, podem ter contribuído para o menor poder discriminante entre essas dimensões. Apesar de os autores da escala já terem sugerido a existência de fatores de ordem superior constituídos a partir dos fatores de primeira ordem presentes no modelo pentafatorial (Fernández-Álvarez et al., 2003), eles defendem manter a distinção conceitual entre as dimensões, tendo em vista tanto a utilidade prática do instrumento quanto a necessidade de haver mais estudos empíricos que permitam uma melhor delimitação do construto.

Ainda a respeito das correlações entre os fatores (Figura 1), observa-se que houve correlações significativas entre as dimensões "Expressão" e "Atenção" $(r=-0,73 ; p<0,01)$, "Expressão" e "Operação" $(r=-0,27$; $p<0,01)$, "Expressão" e "Envolvimento" $(r=0,10$; $p<0,05)$ e "Atenção" e "Operação" $(\mathrm{r}=0,69 ; p<0,01)$. Essas correlações são semelhantes às encontradas por Castañeiras et al. (2008), em uma amostra argentina, e Carvalho et al. (2011), em uma amostra portuguesa. Em conjunto, revelam que a dimensão "Expressão" tende a associar-se negativamente com "Atenção" e "Operação" e positivamente com "Envolvimento", ou seja, quanto mais próxima for a expressão emocional do terapeuta, menos focada será sua atenção e menos planejado será seu estilo de operação. Por sua vez, quanto maior sua expressividade emocional, maior será seu envolvimento afetivo com questões relacionadas ao trabalho. "Atenção" e "Operação" apresentam uma correlação positiva, ou seja, quanto mais focada a atenção, mais planejada será a operação.

\section{Análise da versão reduzida do Questionário do Estilo Pessoal do Terapeuta em relação a tempo de experiência e orientação teórica}

Verificou-se uma correlação entre o tempo de experiência do terapeuta e as dimensões do EPT-C. As correlações foram significativas $(p<0,01)$ para todas as dimensões: "Atenção" $(r=-0,26)$, "Operação" $(r=-0,27)$, "Expressão" $(r=0,13)$ e "Envolvimento" $(r=-0,10)$. Observa-se que, com o aumento dos anos de experiência, a atenção do terapeuta tende a ficar menos focalizada, ou seja, mais ampla. Resultados semelhantes foram encontrados por Oliveira et al. (2006) e Castañeiras et al. (2008). Além disso, seu estilo de operar no setting terapêutico torna-se menos planejado, ou mais espontâneo. Por sua vez, sua expressão emocional torna-se mais próxima, e seu envolvimento emocional com questões relacionadas ao trabalho fora do seu âmbito de atuação tende a diminuir. Essa diminuição pode ter uma função adaptativa, protegendo o terapeuta de possíveis efeitos negativos da sua prática profissional. Nesse sentido, estudos apontam que um envolvimento excessivo com questões do paciente fora do horário de trabalho tende a ser maior no início da carreira e pode ser um preditor de burnout (Skovolt \& Ronnestad, 2003; Steel, Macdonald, Schroder, \& Mellor-Clark, 2015).

Uma ANOVA one-way foi conduzida para identificar as diferenças entre as médias intergrupais do estilo pessoal do terapeuta em função de sua orientação teórica. Testes post hoc (Scheffe e Games Howell) foram conduzidos para especificar a natureza dessas diferenças. A Tabela 4 apresenta as médias e desvios padrão de cada dimensão por orientação teórica.

Tabela 3

Quadrado das Correlações entre os Fatores do EPT-Q

\begin{tabular}{|c|c|c|c|c|}
\hline & Atenção & 2 & 3 & 4 \\
\hline 1. Atenção & - & & & \\
\hline 2. Envolvimento & 0,008 & - & & \\
\hline 3. Expressão & 0,53 & 0,01 & - & \\
\hline 4. Operação & 0,48 & 0,001 & 0,07 & - \\
\hline
\end{tabular}




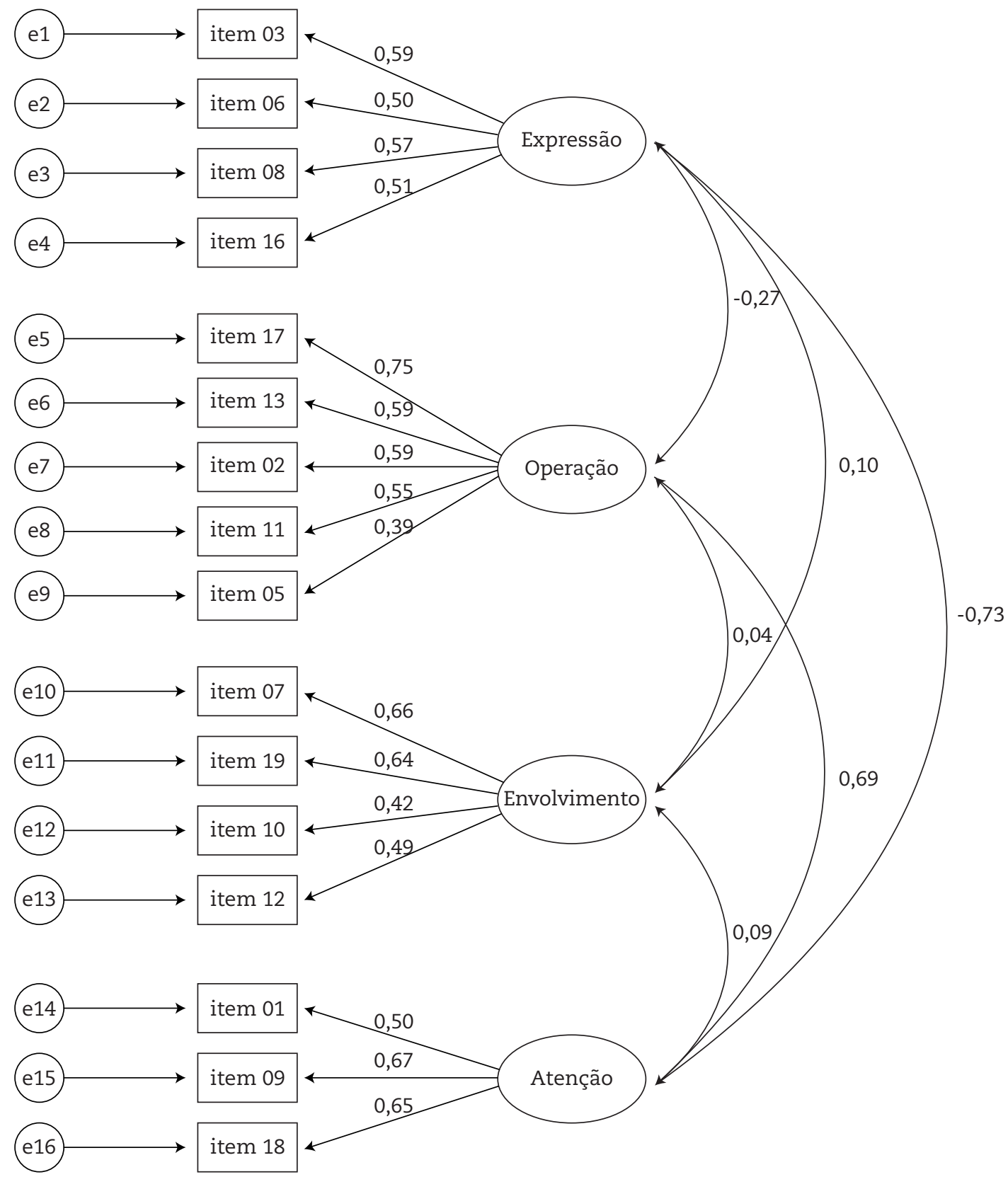

Figura 1. Coeficientes de Covariância e de Cargas Fatoriais Estandardizados Obtidos no Modelo Tetrafatorial

Tabela 4

Média e desvio padrão por dimensão do estilo pessoal do terapeuta e orientação teórica

\begin{tabular}{lcccc}
\hline Orientação teórica & $n$ & Atenção & Expressão & Operação \\
\hline Análise do comportamento & 132 & $3,91(0,90)_{\mathrm{a}}$ & $3,45(0,88)_{\mathrm{a}}$ & $3,09(0,68)_{\mathrm{a}}$ \\
Analítica & 77 & $2,79(0,83)_{\mathrm{b}}$ & $4,13(0,83)_{\mathrm{b}}$ & $2,35(0,66)_{\mathrm{c}}$ \\
Cognitiva & 123 & $3,85(0,90)_{\mathrm{a}}$ & $3,48(0,84)_{\mathrm{a}}$ & $3,51(0,86)_{\mathrm{b}}$ \\
Humanista-existencial & 90 & $2,87(0,97)_{\mathrm{b}, \mathrm{d}}$ & $3,91(0,85)_{\mathrm{b}}$ & $2,09(0,61)_{\mathrm{c}}$ \\
Psicanálise & 170 & $2,08(0,94)_{\mathrm{c}}$ & $4,14(1,07)_{\mathrm{b}}$ & $2,26(0,69)_{\mathrm{c}}$ \\
Sistêmica & 50 & $3,36(0,95)_{\mathrm{d}}$ & $3,68(0,96)_{\mathrm{a}, \mathrm{b}}$ & $2,49(0,81)_{\mathrm{c}}$ \\
Total & 642 & $3,08(1,16)$ & $3,80(0,98)$ & $2,66(0,89)$ \\
\hline
\end{tabular}

Nota: Médias com subscritos iguais na mesma coluna não apresentam diferenças significativas entre si. Foram excluídas das análises aquelas orientações teóricas com menos de 30 participantes por grupo. Não houve diferenças significativas entre as orientações teóricas na dimensão "Envolvimento", tendo sido excluída da tabela. 
A ANOVA indicou diferenças significativas entre os grupos nas seguintes dimensões do EPT-C: Atenção $F(7,666)=59,85, p<0,01$; Expressão $F(7,666)=10,69$, $p<0,01$, e Operação $F(7,666)=49,96, p<0,01$, resultados semelhantes aos encontrados por outros pesquisadores (Castañeiras et al., 2008; Fernández-Álvarez et al., 2003; Oliveira et al., 2006). Convém salientar que, neste estudo, diferentemente de estudos prévios que empregaram o EPT-C, houve maior diversidade de orientações teóricas, permitindo separar terapeutas cognitivos e analistas do comportamento, comumente agrupados na categoria cognitivo-comportamental. Essa separação também foi motivada por recentes debates em torno da diferenciação filosófica e teórica entre as duas abordagens (Wedding \& Corsini, 2013).

Na dimensão "Atenção", as médias dos grupos de analistas de comportamento e terapeutas cognitivos não diferiram significativamente entre si, mas apresentaram diferenças significativas em relação a todos os outros grupos. Além disso, psicanalistas também apresentaram diferenças em relação a terapeutas humanista-existenciais, analíticos e sistêmicos (todos os $p<0,01)$ nessa dimensão. Por fim, terapeutas analíticos e sistêmicos apresentaram também diferenças significativas entre si $(p<0,05)$ quanto à "Atenção". Em linhas gerais, pode-se observar que analistas do comportamento e terapeutas cognitivos apresentam uma atenção mais focada, e os psicanalistas apresentam uma atenção mais aberta, mesmo quando comparados a terapeutas analíticos, sistêmicos e humanista-existenciais.

Na dimensão "Expressão", um padrão semelhante ao anterior foi encontrado. Terapeutas de orientação analítica, humanista-existencial e psicanalítica apresentaram médias significativamente superiores às dos terapeutas cognitivos e analistas do comportamento, indicando que esse primeiro grupo tendeu a enfatizar os aspectos emocionais (e.g., proximidade emocional e expressão das emoções) em sua prática clínica. Os sistêmicos, por sua vez, não diferiram de nenhum grupo nessa dimensão. Esse resultado difere do encontrado por Castañeiras et al. (2008). Nesse estudo, os psicanalistas argentinos apresentaram médias significativamente inferiores às demais orientações teóricas, inclusive humanista-existencial e psicodinâmica. Por fìm, na dimensão "Operação", houve diferenças significativas entre terapeutas cognitivos e os demais grupos, inclusive o de analistas do comportamento. Em relação a essa diferença entre esses dois grupos que são comumente tratados sob a mesma categoria (i.e., cognitivo-comportamental), é provável que esse aspecto operativo seja o mais sensível para detectar as contribuições teóricas e filosóficas na formação do estilo pessoal de terapeutas das duas abordagens. Nele, talvez se expressem de modo mais claro as diferenças de postura filosófica e teórica dos profissionais mediante o comportamento no setting terapêutico.
Ainda sobre a dimensão "Operação", coerente com os estudos de Castañeiras et al. (2008) e Oliveira et al. (2006), terapeutas de orientações psicanalítica, humanista-existencial, analítica e sistêmica, em comparação a terapeutas cognitivos e analistas do comportamento, apresentaram uma menor estruturação do seu manejo técnico (i.e., operação mais livre).

Em conjunto, esses resultados fortalecem o poder do EPT-C em discriminar padrões estilísticos entre os terapeutas de distintas orientações teóricas. De forma geral, terapeutas de abordagens psicanalítica, humanista-existencial, analítica e sistêmica formaram um grupo mais semelhante entre si do que os terapeutas das abordagens cognitiva e analítico-comportamental. Enquanto o primeiro grupo apresentou médias mais baixas em "Atenção" e "Operação" (i.e., atenção mais ampla e operação mais livre), o segundo apresentou médias mais altas nessas mesmas dimensões (i.e., atenção mais focada e operação mais estruturada). No entanto, cabe ressaltar que dentro desses próprios grupos houve diferenças significativas de médias entre os profissionais.

Essas diferenças, que também foram encontradas em outros estudos, podem ser explicadas, em parte, pelos pressupostos epistemológicos, teóricos e técnicos que fundamentam as abordagens e guiam a atuação do terapeuta. Em um estudo sobre os pressupostos epistemológicos presentes na formação de terapeutas de orientações psicanalítica, cognitiva e comportamental, Heffer e Sandell (2009) observaram que, enquanto a formação em psicanálise enfatizava aspectos afetivos e motivacionais (i.e., relacionados à atenção flutuante e ao uso dos sentimentos do terapeuta no manejo de transferência-contratransferência), as abordagens cognitiva e comportamental enfatizavam uma postura mais empírica e racionalista. Essa postura parece estar fortemente associada a aspectos técnicos do estilo pessoal do terapeuta (e.g., operação mais estruturada e busca de informação de forma mais focada). Autores como Arthur (2000), Oliveira et al. (2006) e Lee et al. (2013) parecem estar de acordo com essas asserções e reafirmam a necessidade de se investigar o poder preditivo de variáveis epistemológicas, teóricas e técnicas que estão na base da formação do terapeuta sobre os padrões estilísticos. Nessa perspectiva, estudos futuros poderiam testar, por exemplo, o poder preditivo da orientação epistêmica sobre o estilo pessoal do terapeuta, demonstrando em que medida seus pressupostos epistemológicos básicos podem exercer controle sobre seu comportamento no setting terapêutico.

Conclui-se que a análise fatorial confirmatória do EPT-C revelou uma medida com boas propriedades psicométricas entre os psicoterapeutas brasileiros participantes deste estudo. O modelo tetrafatorial revelou-se mais parcimonioso e com melhores índices de ajuste do que o modelo com cinco fatores originalmente proposto. Isso indica a necessidade de 
revisão das dimensões da medida, uma vez que estudos anteriores já apontavam para a fragilidade do fator "Instrução" em termos de consistência interna, qualidade e quantidade dos itens tanto em sua versão reduzida (Carvalho et al., 2011; Castañeiras et al., 2008) quanto em sua versão original (Oliveira et al., 2006). Além disso, o baixo poder discriminativo entre as dimensões "Atenção", "Expressão" e "Operação" demonstra a necessidade de investigar a medida a partir de modelos que contemplem fatores de segunda ordem, o que já vem sendo sugerido por outros autores (Castañeiras et al., 2008; Fernández-Álvarez et al., 2003). Estudos futuros poderiam testar esse modelo supraordinal em outra amostra de terapeutas.

Este estudo avança ao fornecer mais evidências de validade da medida do estilo do terapeuta em um contexto brasileiro, possibilitando a comparação de diferentes orientações teóricas dentro e fora do país.
Também evolui no sentido de possuir uma amostra considerada grande, o que, diferentemente de outros estudos com o EPT-C, possibilitou a composição de uma maior quantidade subgrupos de terapeutas de diferentes orientações teóricas, com maior número de participantes por grupo. Por fim, o EPT-C demonstrou ser uma medida promissora, cuja utilização pode extrapolar o contexto de investigação do efeito do estilo do terapeuta sobre o tratamento. Sugere-se também testar seu poder de associação com outros construtos da trajetória de desenvolvimento pessoal do terapeuta, como seu estilo epistêmico, suas estratégias de regulação emocional e seu bem-estar. Esse aspecto mais pessoal, e menos técnico, do terapeuta tem recebido considerável atenção nos últimos anos (Orlinsky \& Ronnestead, 2005); nesse contexto, o EPT-C pode contribuir para ampliar o escopo dessas investigações.

\section{Referências}

Arthur, A. R. (2000). The personality and cognitive-epistemological traits of cognitive behavioral and psychoanalytic psychotherapists. British Journal of Medical Psychology, 73(2), 243-257. doi: 10.1348/000711200160453

Baldwin, S. A., \& Imel, Z. (2013). Therapist effects. Em M.J. Lambert (Ed.), Bergin and Garfield's handbook of psychotherapy and behavior change (6 ${ }^{\text {th }}$ ed., pp. 258-297). New York: Wiley.

Byrne, B. M. (2001). Structural Equation Modeling with AMOS: Basics concepts, applications, and programming. New Jersey: Lawrence Erlbaum Associates.

Castañeiras, C., Ledesma, R., García, F., \& Fernández-Álvarez, H. (2008). Evaluación del estilo personal del terapeuta: Presentación de uma versión abreviada del EPT-C. Terapia Psicológica, 26(1), 5-13. doi: 10.4067/S0718-48082008000100001

Carvalho, H., Corbella, S., \& Matos, P.M. (2011). Confirmatory factor analysis of the Portuguese brief version of the Personal Style of the Therapist Questionnaire (PST-Q). Revista Argentina de Clínica Psicológica, 20(1), 79-90. Recuperado de http://www.clinicapsicologica. org.ar/download_file.php?idt $=72 \&$ hax $=2$ THZS41YO

Degnan, A., Seymour-Hyde, A., Harris, A., \& Berry, K. (2016). The role of therapist attachment in alliance and outcome: A systematic literature review. Clinical Psychology \& Psychotherapy, 23(1), 47-65. doi: 10.1002/cpp.1937

Fernández- Álvarez, H., García, F., Lo Bianco, J., \& Santomá, S. (2003). Assessment Questionnaire on the personal style of the therapist PST-Q. Clinical Psychology and Psychotherapy, 10(2), 116-125. doi: 10.1002/cpp.358

Hair, J. F.; Anderson, R.E.; Tatham, R. L., \& Black, W. (2005). Análise multivariada de dados. Porto Alegre: Bookman.

Heffer, B., \& Sandell, R. (2009). The role of learning style in choosing one's therapeutic orientation. Psychotherapy Research, 19(3), $283-292$. doi: 10.1080/10503300902806673

Heinonen, E., Lindfors, O., Harkanen, T., Virtala, E., Jaaskelainen, T., \& Knekt, P. (2014). Therapists' professional and personal characteristics as predictors of working alliance in short-term and long-term psychotherapies. Clinical Psychology and Psychotherapy, 21(6), 475-495. doi: $10.1002 /$ cpp.1852

Lee, J. A., Neimeyer, G., \& Rice, K. G. (2013). The relationship between therapist epistemology, therapy style, working alliance, and interventions use. American Journal of Psychotherapy, 67(4), 323-345. Recuperado de http://www.ingentaconnect.com/content/afap/ ajp/2013/00000067/00000004/art00002

Marôco, J. (2014). Análise das equações estruturais. (2 ${ }^{\mathrm{a}} \mathrm{ed}$.). Lisboa: ReportNumber.

Oliveira, M. S., Nunes, M. L. T., Fernández-Álvarez, H., \& García, F. (2006). Estilo pessoal do terapeuta: dados preliminares da versão brasileira do EPT-Q. Psico, 37(3), 241-247. Recuperado de http://revistaseletronicas.pucrs.br/ojs/index.php/revistapsico/article/view/1445

Orlinsky, D. E., \& Ronnestad, M. H. (2005). How psychotherapists develop: A study of therapeutic work and professional growth. Washington, D.C.: American Psychological Association.

Råbu, M., Moltu, C., Binder, P., \& McLeod, J. (2015). How does practicing psychotherapy affect the personal life of the therapist? A qualitative inquiry of senior therapists' experiences. Psychotherapy Research, 1-13. doi: 10.1080/10503307.2015.1065354

Schöttke, H., Flückiger, C., Goldberg, S. B., Eversmann, J., \& Lange, J. (2015). Predicting psychotherapy outcome based on therapist interpersonal skills: A five-year longitudinal study of a therapist assessment protocol. Psychotherapy Research, 26(1), 1-11. doi: 10.1080/10503307.2015.1125546 
Skovholt, T.M., \& Ronnestad, M.H. (2003). Struggles of the novice counselor and therapist. Journal of Career Development, 30(1), 45-58. doi: 10.1023/A3A1025125624919

Steel, C., Macdonald, J., Schroder, T., \& Mellor-Clark, J. (2015). Exhausted but not cynical: Burnout in therapists working within Improving Access to Psychological Therapy Services. Journal of Mental Health, 24(1), 33-37. doi: 10.3109/09638237.2014.971145

Wedding, D., \& Corsini, R. J. (2013). Current Psychotherapies. 10 $0^{\text {th }}$ ed. New York: Wiley.

Apêndice A

Questionário do Estilo Pessoal do Terapeuta (EPT-Q)

Versão Reduzida

O presente inventário está destinado a conhecer seu estilo pessoal como terapeuta.

Não existem respostas boas ou ruins, já que diferentes estilos podem ser igualmente benéficos. Queremos que responda segundo seja seu modo mais geral e freqüente de levar adiante seu trabalho. Não pense muito no significado de cada afirmação. A resposta mais espontânea é a mais valiosa.

Como anotar:

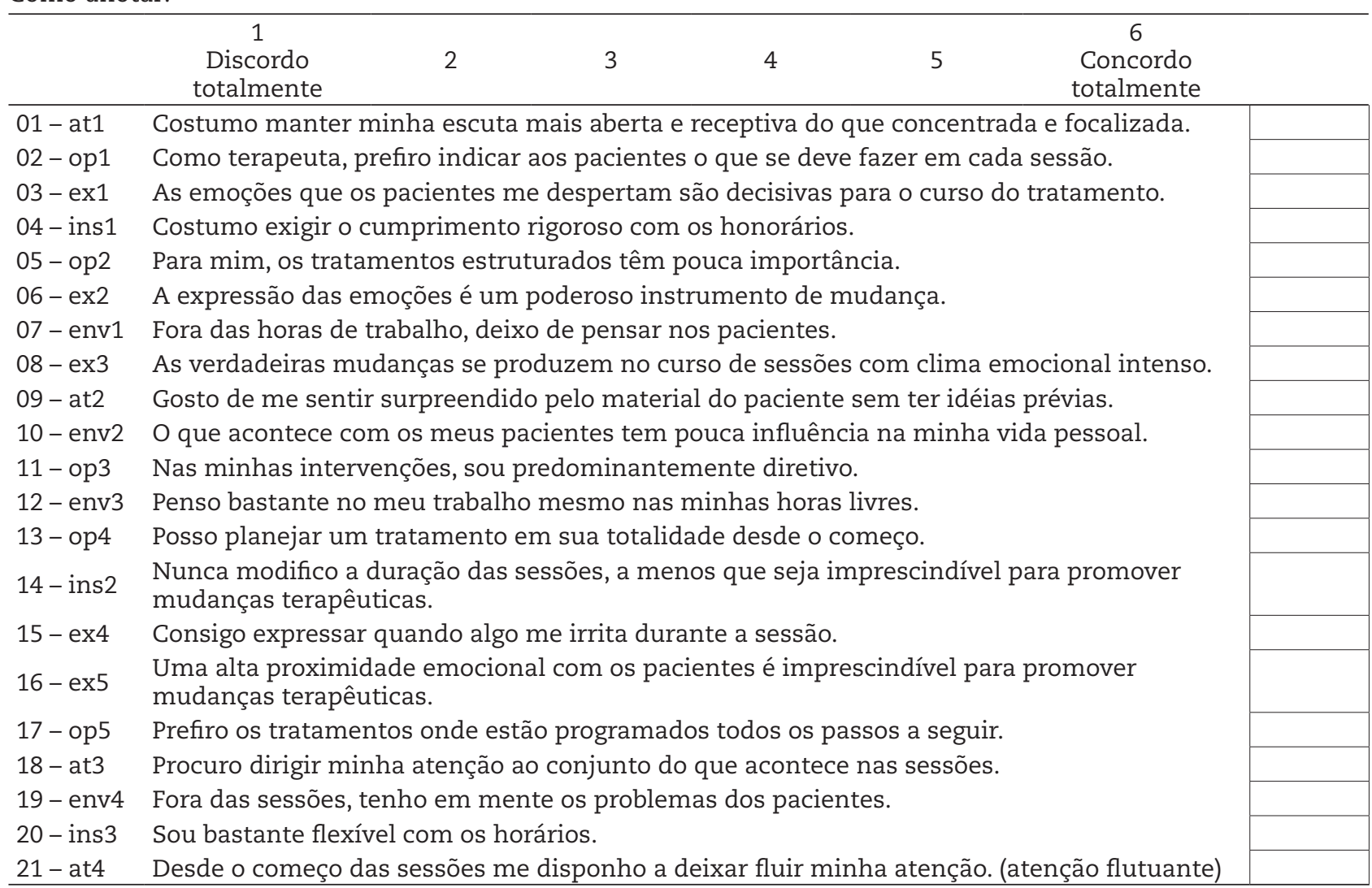

Nota: At=Atenção; Env=Envolvimento; Ex=Expressão; Ins=Instrução; Op=Operação.

\section{Sobre os autores}

Emanuel M. Silva Palma é Psicólogo, Mestre e Doutorando em Psicologia pela Universidade Federal da Bahia.

Sônia Maria Guedes Gondim é Psicóloga pelo Centro de Ensino Superior de Juiz de Fora e possui Doutorado pela Universidade Federal do Rio de Janeiro. Atualmente é Professora Associada Nível IV da Universidade Federal da Bahia. 